\title{
Application of Bundle Intervention in Infant Chloral Hydrate Enema Sedation
}

\author{
Sha Wang, Caiqing Wu*, Shaoxin Lin, Xufen Huang \\ Department of Paediatrics, The First Affiliated Hospital of Jinan University, Guangzhou, China \\ Email address: \\ 378371668@qq.com (Sha Wang),949193073@qq.com (Caiqing Wu) \\ ${ }^{*}$ Corresponding author
}

To cite this article:

Sha Wang, Caiqing Wu, Shaoxin Lin, Xufen Huang. Application of Bundle Intervention in Infant Chloral Hydrate Enema Sedation. American Journal of Pediatrics. Vol. 7, No. 3, 2021, pp. 174-177. doi: 10.11648/j.ajp.20210703.26

Received: August 19, 2021; Accepted: August 31, 2021; Published: September 8, 2021

\begin{abstract}
It is annoying for infants to operate examination, because they often cry during examination, which makes it difficult to carry out the examination smoothly. Therefore we aimed to investigate the effect of bundle intervention on chloral hydrate enema sedation in infants. This observational analytic study was a design of a non-randomized controlled trail, we conducted in infants aged from 0 to 3 who were hospitalized in a pediatric hospital. A total of 293 infants from July to December in 2020 were sedated by chloral hydrate enema. According to the admission time, they were divided into a control group (admitted from July to September) and an experimental group (admitted from October to December). The control group received chloral hydrate enema sedation under conventional nursing, while the experimental group was given both the conventional chloral hydrate enema sedation and bundle intervention. The success rate of chloral hydrate enema sedation was compared between the two groups. The success rate of chloral hydrate sedation in the experimental group was $92.50 \%$, significantly higher than that in the control group (74.60\%), and there was a significant difference between the two groups $(\mathrm{P}<0.05)$. Therefore, bundle intervention can effectively improve the sedation success rate of chloral hydrate enema in infants and relieve the pain caused by repeated enema, which has great clinical application value.
\end{abstract}

Keywords: Bundle Intervention, Infants, Chloral Hydrate Enema, Sedation

\section{Introduction}

In the implementation of Electrocardiogram, Echocardiography, Computed Tomography (CT), Magnetic Resonance Imaging (MRI), and other diagnostic examinations for infants and children, it is often difficult to operate because of crying, making it is necessary to use chloral hydrate enema sedation for a successful examination. Chloral hydrate, chemically named 2,2,2-trichloro-1,1-ethylene glycol, is a hypnotic and anticonvulsant, that enters the human body through oral or intestinal routes. After being metabolized by the organs as such the liver and kidney into active trichloroethane to produce its effects, it will finally be excreted by the kidney making sleepy effects that are similar to physiological sleep state. The hypnotic mechanism is mild and has no significant residual effects [1]. Due to the bitter taste of chloral hydrate, oral administration is often too difficult to cooperate, and the clinical administration of the drug is mostly conducted through the intestinal way. Infants usually fall asleep $10 \sim 20$ minutes after administration, lasting $6 \sim 8$ hours, without any discomfort and accumulative poisoning after waking up [2]. However, increased abdominal pressure, agitation and other reasons after enema may cause drug overflow in some infants, which lead to sedation failure. Repeated administration will increase adverse reactions, nursing workload and diagnosis and treatment difficulties. Bundle intervention incorporates a series of nursing interventions [3, 4], each of which has been clinically proven to be able to improve the symptoms and prognosis of patients. And the joint implementation shows much more clinical effects than any single ones [5]. In this study, 293 infants aged from 0-3 years who were sedated with chloral hydrate were taken as the research objects to explore the application effects of bundle intervention in chloral hydrate enema sedation for infants. 


\section{Objects and Methods}

\subsection{Research Object}

A total of 293 infants who received chloral hydrate enema sedation in the pediatric department of a hospital from July 2020 to December 2020 were selected as the study objects. Inclusion criteria: (1) Hospitalized infants aged from 0 to 3 years. (2) No anaphylactic history of chloral hydrate. (3) Sedation for CT or electrocardiogram rather than disease treatment. (4) Family members of the children were acknowledged of the study purpose and signed the relevant informed consent. Exclusion criteria: (1) Other sedative medication history. (2) Obvious heart diseases, such as congestive heart failure, arrhythmia that cannot be controlled by medication, etc. (3) Lung failure, such as severe pneumonia. (4) Acute and chronic renal failure. 293 infants were divided into the control group (134 cases) and experimental group (159 cases) according to the admission time. The infants admitted from July to September in 2020 were set as the control group, while the infants admitted from October to December in 2020 were the experimental group. The difference in baseline data between the two groups was not statistically significant $(\mathrm{P}>0.05)$, indicating that the two groups were comparable (Table 1). There were 105 males and 54 females in the experimental group, with an age of $(1.84 \pm 0.78)$ years old. In the control group, there were 86 males and 48 females with an age of $(1.76 \pm 0.72)$ years old, and there was no significant difference in basic data between the two groups $(\mathrm{P}>0.05)$.

Table 1. General data of infants with chloral hydrate enema sedation in the two groups.

\begin{tabular}{llllll}
\hline \multirow{2}{*}{ Groups } & \multirow{2}{*}{ Case } & Gender (n) & Year (year, $\overline{\mathbf{x}} \pm \mathbf{s})$ & Weight \\
\cline { 2 - 5 } & & male & female & $\overline{\mathbf{x}} \pm \mathbf{s})$ \\
\hline Experimental group & 159 & 105 & 54 & $1.84 \pm 0.78$ & $10.8 \pm 2.37$ \\
Control group & 134 & 86 & 48 & $1.76 \pm 0.72$ & $11.00 \pm 1.68$ \\
$P$ & & 0.74 & & 0.10 & 0.99 \\
\hline
\end{tabular}

\subsection{Methods}

Both groups received chloral hydrate enema sedation. Control group: during the examination period, the infants were sedated by chloral hydrate enema with routine nursing measures under the doctor's advice. According to the operation procedure of enema, infants were placed in the prone or lateral position, NO. 8 rubber sputum suction tube was selected, and the insertion depth was set according to the age of objectives $(4 \sim 5 \mathrm{~cm}$ for $<1.5$ years, $8 \sim 10 \mathrm{~cm}$ for $1.5 \sim 3.0$ years), $5 \%$ chloral hydrate was slowly injected, and basic daily care was arranged [6]. Experimental group: bundle intervention was conducted under routine care. Before the intervention, we analyzed all clinical data on the failure of chloral hydrate enema sedation under routine care from July to September in 2020 to find out the main influencing factors of chloral hydrate enema sedation and collected a group of bundle intervention measuring methods accordingly: (1) Different catheters were selected according to the individual differences of infants to increase the depth of insertion. No. 8 sputum suction tubes were selected for infants within 12 months, while NO. 12 for infants aged from 12 months and 3 years old, with an insertion depth of $15 \sim 20 \mathrm{~cm}$. At this time, the end of the catheter can reach the lower sigmoid colon, and the large volume of the sigmoid colon reduces the amount of liquid flowing into the rectum, alleviating constipation caused by the stimulation of the rectum, which can prolong the indwelling time of the liquid [7-9]. Besides, increasing the insertion depth can block the chloral hydrate from reaching the lower and middle rectal veins, resulting in the absorption through the superior rectal vein just before entering the liver through the portal vein and being metabolized into active trichloroethane, producing sedation faster with a more satisfactory effect
[10]. (2) Stool emptying. Breastfeeding should be suspended $2 \sim 4$ hours before enema, and the children who did not defecate on the same day were given catharsis. This method can leave the sigmoid colon and rectum in an empty state, and will not raise the pressure in the intestine after enema. Then, the contact area between the gavage fluid and intestinal mucosa will increase, which is conducive to intestinal mucosal absorption and achieve a better sedative effect of enema [11]. (3) Improved halt enema was adopted as previously reported [12]. The anal canal was gently inserted into the anus and then stopped for $3 \sim 5$ seconds, followed by slowly injection of drug solution. Next, the anal canal was reverse folded and pulled out after pinching the buttocks for $3 \sim 5$ minutes. This process continues to maintain the side-lying position with the head low and hip-high. Buttocks were raised by $30 \sim 40^{\circ}$, and avoided vertical holding, to prolong the retention time of the drug solution in the colon and achieve full absorption of the intestinal mucosa. In this way, the drug solution can be prevented from flowing into the rectum to stimulate the anal sphincter and cause the defecation reflex, affecting the sedation effect of enema $[13,14]$. (4) Sleep deprivation. Before the enema, the nurse evaluated the basic situation of the infant and set the time of sleep deprivation according to the situation as well as an appointment to check the child in the morning. The parents were instructed to let the child sleep $1 \sim 2$ hours later than usual the previous night and get up $1 \sim 2$ hours earlier than usual in the morning. To make an appointment in the afternoon for examination or treatment, children should not take napping and keep them awake until examination or treatment. Through sleep intervention, children are deprived of part of the normally required amount of sleep reducing the level of awakening, and can quickly enter the sleep state after using chloral hydrate enema $[15,16]$. 


\subsection{Observation Indicators}

The sedative effects of one-time medication in the two groups were analyzed: 30 min after the medication, the infants fell asleep quietly, without agitation and liquid overflow, and the follow-up examination could be completed successfully, which was judged to be successful. On the other hand, the children were still excited or agitated, so that the examination could not be completed was considered a failure.

\subsection{Statistical Analysis}

All data were statistically analyzed by SPSS 24.0 statistical software. Measurement data conforming to normal distribution were represented by $\overline{\mathrm{x}} \pm \mathrm{s}$ and $\mathrm{t}$-test was performed. Counting data were tested by $\mathrm{x}^{2}$ test, and $\mathrm{P}<0.05$ was considered statistically significant.

\section{Results}

The sedation effect of the two groups was as follows: the total effective rate of the experimental group was $92.50 \%$ (147/159), while that of the control group was $74.60 \%$ $(100 / 134)$. There were significant differences between the two groups $(\mathrm{P}<0.05)$ (Table 2$)$.

Table 2. Comparison of results between two groups of infants with chloral hydrate enema sedation.

\begin{tabular}{llll}
\hline Groups & Number & Success & Failure \\
\hline Experimental group & 159 & $147(92.5)$ & $12(7.5)$ \\
Control group & 134 & $100(74.6)$ & $34(25.4)$ \\
$x^{2}$ & & 17.46 & \\
$P$ & & 0.00 & \\
\hline
\end{tabular}

\section{Discussion}

Compared with other hypnotic drugs, chloral hydrate has a quick hypnotic effect and a lower impact on the sleep phase [17]. It can cause approximately normal physiological sleep activities, and no drowsiness, fatigue, and other residual effects after waking up, and it is safe to give the drug by enema. However, it has been reported that the traditional chloral hydrate enema has a low success rate within infants under one year old, and the rate is as high as $51.9 \%$. Repeated or large dose administration may increase drug toxicity, decreasing drug safety [1, 18], and causing dissatisfaction among family members, and increasing the workload of medical staff. Bundle intervention mainly refers to a comprehensive nursing intervention scheme that incorporates various nursing methods which have been proved to be effective at present according to the evidence-based clinical practice. Studies have shown that bundle intervention is a structured method, which is of great significance for improving the quality of medical work and the outcome of patients' treatment $[19,20]$. In this study, bundle intervention was applied to chloral hydrate enema sedation in children to explore its clinical application value. We used the evidence-based PICO system to review the literature, and selected literature with high-level evidence, appropriate clinical situation, and patient population, and summarized the following bundle interventions: (1) Individualized catheter selection and increased insertion depth. (2) Stomach emptying before enema. (3) Using the improved halt enema method. (4) Individualized sleep deprivation. The results of this study showed that the success rate of chloral hydrate enema sedation in the experimental group was higher than that in the control group, indicating that the intervention measures significantly improved the success rate of chloral hydrate enema sedation. In conclusion, the application of bundle intervention in chloral hydrate enema sedation in infants can effectively improve the success rate of sedation and make the operation more standardized and scientific, which is worthy of promotion and research in various clinical fields.

\section{Conclusion}

This study showed bundle intervention can effectively improve the sedation success rate of chloral hydrate enema in infants and relieve the pain caused by repeated enema, which has great clinical application value.

\section{References}

[1] Cui Y, Guo LT. Comparison of sedative efficacy of chloral hydrate in pediatric patients of different ages undergoing non-invasive diagnostic procedures [J]. Chin J Anesthesiol, 2021 (01): 85-87.

[2] Li M, Pang LM, Yang SJ, et al. Nursing observation of infant chloral hydrate retention enema $[\mathrm{J}]$. Jilin Medical Journal, 2009 (16): 1779-1780.

[3] CHABOYER W, BUCKNALL T, WEBSTER J, et al. The Effect of a Patient Centred Care Bundle Intervention on Pressure Ulcer Incidence (INTACT): A Cluster Randomized Trial [J]. International Journal of Nursing Studies, 2016, 64: 63-71.

[4] JIMÉNEZ-MARTÍNEZ E, CUERVO G, CARRATALÀ J, et al. A Care Bundle Intervention to Prevent Surgical Site Infections After a Craniotomy [J]. Clinical Infectious Diseases, 2020, 72 (9): 1686-1686.

[5] MAO Z, MIN X, RUOYU H, et al. Bundles of care for prevention of ventilator-associated pneumonia caused by carbapenem-resistant Klebsiella pneumoniae in the ICU [J]. American journal of translational research, 2021, 13 (4): 3561-3572.

[6] Cui Y, Yang SF. Pediatric Nursing [M]. $6^{\text {th }}$ edition. Beijing: People's Medical Publishing House, 2018: 159.

[7] Zhou J, Wang Q, Su CX. Influence of Intubation Depth of Retention Enema on the Enema Retention Time in the Intestine: A Meta-analysis [J]. Nurs J Chin PLA, 2017 (11): 1-7.

[8] Liu M, Cui JM, Liu SM, Liu F, Liu QM, Qian XL. Comparison of the effect of different depths of pediatric enema [J]. Chin J Prac Nurs, 2004 (12): 44.

[9] Nian XH. Nursing Care of Children's Chloral Hydrate Retention Enema [J]. Guide of China Medicine, 2017 (23): 224. 
[10] PUROHIT T J, HANNING S M, WU Z. Advances in Rectal Drug Delivery Systems [J]. Pharmaceutical Development and Technology, 2018, 23 (10): 942-952.

[11] Li XP, Li Y, Yao M. Related research on improving the effect of sedation and hypnosis by nursing intervention methods before chloral hydrate in infants of $0-3$ years old [J]. Chinese Remedies \& Clinics, 2019 (06): 1005-1006.

[12] Li J, Wu XP. The effect of modified chloral hydrate retention enema on the sedative effect of young children [J]. TODAY NURSE, 2015 (06): 132-133.

[13] Liu ZY, Liu M. Application of chloral hydrate in infants and young children's sedative administration method [J]. Chin J of Clinical Rational Drug Use, 2015 (29): 84-85.

[14] Zhang HP, Zhao HL, Dai LF, et al. The effect of modified chloral hydrate retention enema combined with sedation assessment tools on the sedative effect of infants and young children [J]. Electronic Journal Of Practical Clinical Nursing Science, 2020 (22): 197-198.

[15] Gan BH, Shen Q, Lin YE. Influence of Sleep Deprivation Combined with Chloral Hydrate Sedation for Improvement of
Success Rate of Infants and Young Children with MRI Examination [J]. World Journal of Sleep Medicine, 2019 (12): $1712-1714$

[16] Wang Q, Ren XY. Analysis of the effect of sleep deprivation in sedative application of chloral hydrate in infants $[\mathrm{J}]$. J Nurs Train, 2010 (16): 1491-1492.

[17] GUMUS H, BAYRAM A K, POYRAZOGLU H G, et al. Comparison of Effects of Different Dexmedetomidine and Chloral Hydrate Doses Used in Sedation on Electroencephalography in Pediatric Patients [J]. Journal of Child Neurology, 2014, 30 (8): 983-988.

[18] Jin LH, Xu WY, Bai J, et al. Evaluation of efficacy and safety of chloral hydrate sedation for imaging test in children [J]. J Clin Anesthesiol, 2013 (07): 678-680.

[19] Liu HY, Zhang J. Application of cluster management mode in the management of enteral nutrition pump [J]. Shanxi Med J, 2017 (14): 1759-1761.

[20] Kong Y. Application of Cluster Nursing Intervention in the Nursing of Severe Stroke Enteral Nutrition Related Diarrhea [J]. Chin Gen Prac, 2019, v. 22 (S2): 197-199. 